\title{
SPIN, THE GREAT INDICATOR OF VALENCE BEHAVIOUR
}

\author{
J. H. VAN Vleck
}

Harvard University, Cambridge, Massachusetts

\begin{abstract}
This paper opens with a comparison of the electron shell model of Langmuir with the assignment of quantum numbers made by Bohr and slightly modified by Stoner in the years immediately preceding quantum mechanics. A discussion is given of the role of spin in the development of quantum mechanics, culminating in the relativistic Dirac equations. Because of the exclusion principle spin is the great indicator of valence behaviour. This is shown particularly clearly by a vector-model formula first derived by Dirac. The Heitler-LondonSlater-Pauling and Hund-Mulliken approaches are compared. The two methods each have their difficulties but there is often comforting agreement in their conclusions. The results are displayed of some early calculations of binding energies of the hydrogen molecule and of resonance energies.

The final portion of the paper discusses the development of the crystal field theory of the paramagnetic susceptibilities of salts of the iron group during the first decade of quantum mechanics, and how this theory furnishes the key to the theoretical inorganic chemistry underlying the diverse behaviour of the various ions in this group.
\end{abstract}

\section{INTRODUCTION}

By some chance I remember after over thirty five years Enrico Fermi remarking to me, obviously somewhat with tongue in cheek, that a really good theoretical physicist can obtain right answers even with wrong formulas'. On reading Langmuir's paper on valence theory ${ }^{1}$ whose semicentennial is being celebrated by the present symposium, I am tempted to say in the same vein 'a really good theoretical chemist can obtain right answers with wrong models'.

The historian of physics distinguishes between the period of the quantum theory often disparagingly called the 'old quantum theory', and the era of true quantum mechanics. The former embraced roughly the first quarter of the present century, starting with Planck's introduction of his constant $h$ in his radiation formula and terminating when the true ground rules of atomic dynamics were discovered by Heisenberg and others in 1925.

By the same token, one can try to distinguish between the eras of the quantum theory of valence and the quantum mechanics of valence. However, the period of any substantial quantum theory of valence extends over only five years in the first half of the 1920s. Langmuir's theory and the still earlier 


\section{J. H. VAN VLECK}

models of Lewis and Kossel were semi-phenomenological ones, combinations of empiricism and chemical insight. They cannot, however, be said to be quantum theories as they did not utilize the value of Planck's constant, or even use the quantum concept except insofar as it was implicit in the assumption that the atom housed electrons in a discrete number of clls.

\section{BOHR VIS-A-VIS LEWIS AND LANGMUIR}

The historian of valence theory, if he is a chemist rather than physicist, is prone to overlook a paper which Niels Bohr' ${ }^{2}$ wrote in 1921 entitled 'On the structure of the physical and chemical (sic) properties of the elements' (not to be confused with his pioneer paper of 1913 on the hydrogen atom). The name of Bohr is not, for instance, found in the name index of Pauling's classic book on The Nature of the Chemical Bond. In his 1921 paper, Bohr correctly anticipated quantum mechanics (with a few minor exceptions) in the assignment of principal quantum numbers as one progresses from one element to another through the periodic table, including the transition and rare earth series. Figure 1 makes clear the difference between the theories of Langmuir and Bohr. Both make the inert gases come at the right places, but

\begin{tabular}{|c|c|c|c|c|c|c|c|c|c|c|c|c|c|c|c|c|c|}
\hline 0 & 0 & & & & & & & \multicolumn{10}{|c|}{ Langmuir, 1919} \\
\hline $\begin{array}{l}x \\
V\end{array}$ & $\begin{array}{l}X \\
V\end{array}$ & $\begin{array}{l}X \\
V \\
V\end{array}$ & $\begin{array}{l}\bar{X} \\
V\end{array}$ & $\begin{array}{l}x \\
v\end{array}$ & $\begin{array}{l}X \\
V\end{array}$ & $\begin{array}{l}x \\
V\end{array}$ & $\begin{array}{l}X \\
V\end{array}$ & & & & & & & & & & \\
\hline$\Delta$ & $\Delta$ & $\Delta$ & $\bar{\Delta}$ & $\Delta$ & $\Delta$ & $\Delta$ & $\Delta$ & $\Delta$ & $\Delta$ & $\Delta$ & $\Delta$ & 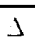 & $\Delta$ & $د$ & $\Delta$ & $\Delta$ & $\Delta$ \\
\hline
\end{tabular}

\begin{tabular}{|c|c|c|c|}
\hline$n=1$ & $\begin{array}{ll} \\
0\end{array}$ & \multicolumn{2}{|c|}{ Bohr, 1921} \\
\hline$n=2$ & $\begin{array}{l}x x \\
x x^{s}\end{array}$ & $\begin{array}{l}x \times \\
x \times p \\
\end{array}$ & \\
\hline$n=3$ & $\begin{array}{l}\vee \vee \Delta \\
\vee \vee \Delta\end{array}$ & $\begin{array}{l}V \vee \Delta \\
V \vee \Delta p\end{array}$ & $\begin{array}{l}\Delta \Delta \Delta \\
\Delta \Delta \Delta\end{array}$ \\
\hline$n=4$ & $\begin{array}{ll}\Delta \Delta_{s} \\
\Delta \Delta^{\prime}\end{array}$ & $\begin{array}{ll}\Delta & \Delta p \\
\Delta & \Delta\end{array}$ & \\
\hline & & & $\begin{array}{r}O-E \\
O X- \\
\text { OXV- } \\
\text { OXVA - }\end{array}$ \\
\hline
\end{tabular}

Figure 1. The progressive filling of Langmuir 'cells' and Bohr orbits. For simplicity, the comparison is carried only through $Z=36(\mathrm{Kr})$. Also shown is the revision of Bohr's assignment of azimuthal quantum numbers which was made by Stoner and which led Pauli to the exclusion principle.

in particular there is considerable difference in how the long periods are developed, the respect in which the Langmuir theory was more pretentious and extensive than the earlier one of Lewis which stressed mainly the electron pair bond and the octet. Nevertheless historians can cite many respects in which Lewis and Langmuir intuitively anticipated many of the results of quantum mechanics. Langmuir, in particular, never allowed more than two 
electrons to a cell, a sort of premonition that the Pauli exclusion principle allows only two electrons to an orbital state. Critics on the other hand can point out that he had his two innermost cells filled to only half of capacity.

As a matter of fact, it is not difficult to recast Langmuir's model to conform to Bohr's assignment of quantum numbers to the various electron sequences. In a footnote to a paper ${ }^{3}$ written in 1926 in another connection, Langmuir does casually mention that Bohr has somewhat different shell structures but observes that his theory could be adapted to Bohr's ideas with 'only a slight modification of the wording' that 'does not otherwise affect the validity of the conclusions'. In retrospect it is surprising that there is practically no literature in the early 1920 s spelling out such an adaptation explicitly. The one instance I am aware of is a book ${ }^{4}$ by Main Smith, too often overlooked.

The approaches of Lewis or Langmuir and Bohr were in some ways quite different. Bohr kept his eye on the facts of atomic spectra, and Langmuir did on the nature of the chemical bond. Lewis and Langmuir stressed stereochemical aspects of valence, and how a completed shell should have essentially cubic symmetry. Lewis appears to have been a hold-out for the atom with static electrons, something painful to the physicist because of Earnshaw's theorem whereas Langmuir was torn between the dynamic and static approaches. The success of the Bohr theory of atomic spectra demanded the dynamic atom, whereas the directional properties of stereochemistry seemed more amenable at the time to a static approach. In his celebrated 1919 paper, Langmuir postulated rather non-committally that the electrons could be either stationary or rotate, revolve or oscillate about definite positions in the atom'. In one subsequent paper he suggested replacing centrifugal force by an equivalent static force ${ }^{5}$, while in another he made elaborate calculations with a particular dynamical model of the helium atom $^{6}$.

\section{THE EXCLUSION PRINCIPLE AND SPIN}

The theory of valence in the old quantum theory reached its heyday in 1925 , with the advent of the Pauli exclusion principle early in that year ${ }^{7}$, and the Uhlenbeck-Goudsmit ${ }^{8}$ proposal of electron spin in its final months. A certain rationale, in terms of quantum numbers, was thus given to the structure of the Mendeléef table, but the large dependence of the energy on spin alignment in the coupling between non-equivalent orbits-for example, the large separation between singlet and triplet states - still was an enigma at the end of 1925 . The successes achieved by the old quantum theory of valence were all qualitative. It is true that in the early 1920 s one did have spectroscopic determinations of heats of dissociation, and rotational and vibrational spectra of molecules were proving susceptible to interpretation, but nothing quantitatively worthwhile came out concerning the theoretical calculation of the strength of chemical bonds. The state of the subject is indicated by the index of the book I wrote in $1925^{9}$ appraising the state of quantum theory as of that period. The index to 'molecule' said 'see hydrogen molecule'; the only one for which the ill-fated calculations were even attempted. 


\section{THE ADVENT OF QUANTUM MECHANICS}

The birth of quantum mechanics changed everything almost overnight. The year 1975, i.e. the year of the third IUPAC congress following the present one, should be a gala one for celebrations. It will be the semicentennial of the discovery of quantum mechanics, and the bicentennial of the American Revolution. In both cases there is some question whether the festive year should be 1975, or 1976, a year later. Matrix mechanics was first introduced in 1925, but Schrödinger did not publish his paper on wave mechanics until 1926, and it was in 1926 that the equivalence of the matrix and wave versions was demonstrated. Correspondingly, the first shots of the Revolution were fired on 19 April 1775, but the Declaration of Independence was voted on 4 July 1776. The type of analogy I am making cannot, however, be pursued too far. The advent of quantum mechanics solved the problem of extranuclear atomic and molecular dynamics for all time, but I often think how nice it would be if there were one commonwealth of the English-speaking nations.

Naturally I am honoured that this plenary session is occasioned by the semicentennial of the papers of Langmuir and Lewis, but I am sure you all know that it was in continental Europe that the true quantum mechanics was discovered, both in the matrix and wave forms. Nevertheless, speaking here in the British Commonwealth it seems particularly appropriate to call attention to important contributions of British scientists in the 1920s. In a 1924 paper just before the dawn $\dagger$ of quantum mechanics, Stoner ${ }^{10}$ formulated correctly how the electrons of given principal quantum number were distributed among states of different azimuthal, and inner quantum numbers, whereas Bohr had the wrong distribution. The distinction in their partitioning is exhibited in our Figure 1. For instance for the shell completed at neon, Stoner had (in present day notation) $1 s^{2} 2 s^{2} 2 p^{6}$ whereas Bohr had $1 s^{2} 2 s^{4} 2 p^{4}$. Furthermore, using empirical rules previously developed by Landé ${ }^{11}$, Stoner had the correct statistical weight, i.e. correct number of Zeeman levels for each value of the inner quantum number. He came within a hair's breadth of getting the exclusion principle - he failed to note that each of his states was inhabited only once.

In 1926 a British physicist, L. H. Thomas ${ }^{12}$, cleared up a factor two that was apparently wrong in the spin-orbit coupling, a difficulty that caused Pauli to talk Kronig out of publishing a paper proposing electron spin prior to Uhlenbeck and Goudsmit. The explanation was a rather recondite relativistic precessing effect. Then, above all, in 1926 and 1928 Dirac made monumental contributions in perfecting quantum mechanics.

In a 1929 paper Dirac ${ }^{13}$ wrote some classic sentences which I often quote: 'The general theory of quantum mechanics is now almost complete, the imperfections that still remain being in connection with the exact fitting in of relativity ideas... The underlying laws necessary for the mathematical theory of a large part of physics and all of chemistry are thus completely known'. In my opinion Dirac was over-modest, for his celebrated 1928 paper $^{14}$ on the quantum theory of the electron solved many of the difficulties

$\dagger$ A little earlier and independently of Stoner, Main Smith had a partitioning scheme in many respects similar to Stoner's, but erred in the assignment of the azimuthal quantum number so that it could not have been used as a basis for the exclusion principle. 
connected with relativity. The previous Klein-Gordon wave equation had the requisite relativistic invariance, but was a rather shabby affair, lacking elegance because solutions of the wave equations belonging to different energies were not orthogonal. Dirac's quantum theory of the electron ${ }^{14}$, one of the most brilliant intellectual achievements of all times, avoided this difficulty, and what is more, all the properties of electron spin came out automatically, and almost magically, like rabbits out of a magician's hat. Uhlenbeck and Goudsmit had already introduced electron spin in late 1925, accidentally practically simultaneously with the advent of the Heisenberg matrix mechanics, but until Dirac's 1928 paper, it was an added ingredient not necessarily built into quantum mechanics. In a certain sense all of chemistry is the child of relativity, for in a Newtonian world spin wouldn't be necessary for an elegant mathematical theory, worthy of the Deity governing the universe. The Pauli principle would then allow only one electron to an orbital state, there would be no electron pairs, and chemistry, in fact, the universe would be very different. Speculations like this I would call only metachemistry, by analogy to metaphysics.

The only courses I ever had in chemistry were as a summer school student at the University of Minnesota exactly 52 and 51 years ago, and so perhaps I should disqualify myself from speaking at a programme reviewing only the last fifty years. In any case, I can mention that I only got as far as qualitative analysis, in the routine courses of those early days, and I never took quantitative analysis, with its terrifying amount of laboratory work. This circumstance I will take as precedent for my analysing for the most part only qualitatively what has been accomplished in the quantum mechanics of valence behaviour, and leave to younger speakers the quantitative study of what has been accomplished in calculating binding energies with the aid of elaborate computing machines. In consequence my attention will be focused primarily on developments prior to 1939. I will show that even though the broad fundamental mathematical principles of quantum chemistry were already known in 1926, it took about a decade for even the qualitative implications of quantum mechanics for valence behaviour to be appreciated and recognized. This unbelievably slow rate of progress was a consequence of there being few workers in the field, as the sudden outburst of quantum mechanics in 1926 found most chemists of the time without the mathematical preparation required for the assimilation of the new discipline, and mathematical physicists were usually ignorant of anything but the most elementary chemistry. For this reason, the applications of quantum mechanics to problems of pure physics, such as spectra, collision phenomena etc. were achieved more rapidly than those to chemistry. To remedy the deficiency many books with titles such as 'quantum chemistry' were written in the 1930 s, but practically none with names such as 'quantum physics', although such terminology would make more sense inasmuch as many branches of physics, such as acoustics, can be handled by classical mechanics, but in chemistry this situation is much less true. Most of the space in such books, as a matter of fact, was devoted to developing the general mathematical structure of quantum mechanics, without anything specifically chemical. Today, in. 1969, the gulf between mathematical physics and chemistry has 
been eliminated by the simple expedient of chemistry departments annexing whole areas of physics, notably molecular spectra. The astrophysicists seem to be those today most interested in atomic spectra, and the electrical engineers those in solid state physics. So an extra-nuclear physicist is almost a man without a country in a physics department.

\section{SPIN AS INDICATOR}

Although it took a decade for all the qualitative repercussions of quantum mechanics on valence theory to be appreciated, certain triumphal features emerged right at the beginning in 1926. One of them was that it explained why spin was an indicator for valence behaviour.

I must confess I remember hardly anything from the chemistry courses I took over 50 years ago. They were pretty much of the cookbook variety and have practically no rapport with the chemical implications of my research over the years. About the only thing I remember from the early courses was that acids turned litmus red and bases turned it blue, and that this paper was a so-called 'indicator' of the chemical state of affairs without having ipso facto any effect on the chemical reaction. This is what has led me to the title of my present paper, for spin has usually a negligible direct effect on a chemical bond, but indicates simply what is going on. The direct electromagnetic forces arising from spin are far too weak to be responsible for chemical bonding, unlike the Parsons ring magneton of 1915 where they were supposed to be enormous. In other words if say the velocity of light were made a thousand times smaller than it really is, and the magnetic moment of the electron correspondingly reduced, the science of chemistry would be virtually the same as it is now, except for a few minor subjects, such as for example ortho-para conversion. And yet at the same time spin is at the heart of chemistry. This is, of course, because of the constraints required by the Pauli exclusion principle. When this principle was first proposed in 1925 it was a purely empirical rule, but it acquired a physical basis and a flesh and blood significance in 1926 when Dirac and Heisenberg ${ }^{15}$ independently showed that it was equivalent to the requirement that the total wave function be antisymmetric when spin is included. Consequently Dirac's classic dictum regarding the mathematical foundations of chemistry which I have quoted could have been written in 1926 instead of 1929 . Still unanswered, and probably it never will be, is why the Creator allows only antisymmetric wave functions.

It is the requirement of antisymmetry that takes the mystery out of why spin is the great indicator of valenœ behaviour, for when two electrons nestle in the same molecular orbital and the valence is saturated their spins have to be antiparallel. On the other hand most molecules with an odd number of electrons have a paramagnetic susceptibility given by the 'spinonly' formula

$$
\chi=N \beta^{2} S(S+1) / 3 k T, \quad(\beta=h e / 4 \pi m c)
$$

with $S=\frac{1}{2}$. The occurrence of paramagnetism was known as a test for free radicals with unsaturated valences even in pre-quantum mechanical days, but equation 1 puts things on a quantitative basis. As an example I might 
SPIN, THE GREAT INDICATOR OF VALENCE BEHAVIOUR

mention that in $1930 \mathrm{I}$ was suspicious of the then existing experimental data according to which the susceptibility of $\mathrm{NO}_{2}$ had less than one fifth the value to be expected from equation 1. At my suggestion Havens ${ }^{16}$ at Wisconsin made new measurements, which agreed with 1 . The trouble with the earlier experimenters was that they had not estimated correctly the degree of dimerization. In fact, Havens's measurements furnished an independent confirmation of a formula for the degree of dimerization proposed by Verhoek and Daniels ${ }^{17}$.

In the early days one could detect free spins only through susceptibility measurements, but since 1945 one has of course the enormously more sensitive tool of paramagnetic resonance, whereby minute amounts of free spins can be detected, permitting study of impurities and lattice defects, abnormal ions caused by radiation damage, and migration of colour centres. The mere observation of electron spin resonance shows the existence of a free radical, but one can do much better than this. From the frequency of the resonance line and its hyperfine structure one can identify where the free spin is located. Magnetic resonance even finds its way into biophysics and astronomy. All this sort of thing is outside the early period being stressed in the present paper, and I will cite only two examples. When the macromolecule of DNA, the double helix of Crick and Watson, is damaged by radiation, it annexes a hydrogen ion from the surrounding moisture. Electron spin resonance not merely shows this is so, but by comparison with the corresponding spectrum of thymine tells us exactly where this proton is attached ${ }^{18}$. In view of Australian pre-eminence in radioastronomy, I should not omit mentioning that electron spin serves as an indicator of where hydrogen is located in galactic space, for the celebrated $21 \mathrm{~cm}$ line of hydrogen, first observed by Ewen and Purcell ${ }^{19}$ at Harvard, arises from the hyperfine structure coupling the electronic and nuclear spins.

When the valences are saturated, materials are usually diamagnetic, precluding use of electron spin resonance. but one can then employ nuclear magnetic resonance. The nuclear spin resonance frequencies serve as an indicator of where the nucleus is located. You all know that a nuclear magnetic resonance spectrometer is now standard equipment for any organic chemistry laboratory. This is again a post-1945 development, not, however, in basic theory but in experimental teohnique.

\section{CENTROSYMMETRIC STATISTICAL CHARGE DISTRIBUTIONS}

Another initial triumph of quantum mechanics for chemistry, besides the spin-indicator property just discussed, is the fact that completed shells of given azimuthal quantum number have a spherically symmetric charge distribution. This symmetry is expressed mathematically in the identity

$$
\sum_{m=-l}^{l}\left|Y_{l}^{m}(\theta, \varphi)\right|^{2}=(2 l+1)
$$

where the $Y_{l}^{m}$ are spherical harmonics normalized to unity. A rationale was thus given to the three-dimensional static atoms of cubic symmetry so dear to the hearts of Lewis and Langmuir. In general the taking of expectation values in quantum mechanics endows the atom with pseudostatic properties. 


\section{J. H. VAN VLECK}

In retrospect it is clear that chemists did not have at the time the spirit of exultation that they should have had over the stereochemical consequences of equation 1 t. The perfect symmetry of completed atomic shells is far greater than cubic and greater than any one dared dream of in earlier days. An immediate explanation is given of why closed shells are diamagnetic. The case of the helium atom is particularly striking-both the models of Langmuir and Bohr had preferred axes, i.e. an atomic quadrupole moment instead of the spherical symmetry we now know goes with $s$ states. The azimuthal quantum number $l$ entering in the new quantum mechanics is one unit lower than $k$ used in the old quantum theory. This fact is fortunate for the following reason. In the old theory the state $k=0$ was arbitrarily excluded because it required the orbit to be a straight line going through the nucleus. The magnetic quantum number quantizing the spatial orientation of the orbit was assumed to have the values $\$$

$$
m_{k}=-(k-1), \ldots, k-1
$$

simply in order to make the number of Zeeman components accord with experimental facts, and the exclusion of $m_{k}= \pm k$ was an ad hoc procedure. Without it the Pauli principle would not have worked. Now, of course we know that instead

$$
m_{l}=-l, \ldots, l
$$

a relation which is of course equivalent to 3 inasmuch as $l=k-1$.

\section{THE HEITLER-LONDON AND HUND-MULLIKEN APPROACHES}

As you all know, the two simplistic formulations, or starting points for deducing valence behaviour from quantum mechanics are respectively the Heitler-London-Slater-Pauling and Hund-Mulliken approaches, based respectively on electrons localized to particular atoms and uncorrelated itinerant electrons whose paths are called molecular orbitals. Of these two methods that of molecular orbitals is by and large the more flexible and powerful, at least from a qualitative standpoint, but I shall focus more attention on the localized approach, partly because it was more emphasized in the early days of quantum mechanics but mainly because with Professor Mulliken on the programme it would be superfluous, even lèse majesté for me to discuss molecular orbitals in depth $\S$.

\footnotetext{
† I did not, for instance, stress this feature strongly enough in a talk on 'The New Quantum Mechanics' that I. gave at a meeting of the American Chemical Society in 1928, published in Chem. Rev. 5, 467 (1928).
}

$\ddagger$ Besides $n, k, m_{\mathrm{k}}$ (or $\left.n, l, m_{1}\right)$ there is also the fourth quantum number $m_{\mathrm{s}}= \pm \frac{1}{2}$ associated with space quantization of the spin. Historically Stoner and Pauli used the set of quantum numbers $n, k, j, m_{\mathrm{j}}$ but in formulating the exclusion principle it is more straightforward to use the $m_{1}, m_{\mathrm{s}}$ system in which spin and orbit are decoupled, as Goudsmit pointed out in what is probably the last important paper of the great numerological period of the old quantum theory that preceded quantum mechanics Z. Phys. 32, 794 (1925).

$\S$ By 1936, Mulliken had already published what Penney and Kynch appropriately called a 'formidable series of papers extending over several years' in a review article they wrote that year, Annu. Rep. Chem. Soc. 33, 37 (1936). 


\section{SPIN, THE GREAT INDICATOR OF VALENCE BEHAVIOUR}

Since $s$ states have such beautiful centrosymmetric properties it was natural that the first efforts with the Heitler-London theory ${ }^{20}$ should employ the simplifying assumption that all valence electrons are effectively in $s$ states. Then the valence behaviour depends entirely on spin rather than orbital orientation. The formulation of the mathematical secular problem connected with the coupling together of the spins in such a model has a rather curious early history. First Heitler ${ }^{2 \mathrm{i}}$ tackled the problem by employing the rather intricate theory of the permutation group. Then Born ${ }^{22}$ wrote a paper showing how the calculations could be simplified by means of Slater determinants. In the meantime Dirac ${ }^{13}$ showed that exchange coupling, which is the cement responsible for the chemical bond in the Heitler-London theory, is equivalent to a coupling

$$
V_{i j}=-\frac{1}{2}\left(1+4 s_{i} \cdot s_{j}\right) J_{i j}
$$

between the spin vectors $s_{i}, s_{j}$ of electrons $i$ and $j$. The corresponding exchange integral is denoted by $J_{i j}$. Instead of recondite group theory or bulky determinants the entire interaction problem becomes essentially that of the vector model so dear to the heart of the atomic spectroscopists. A large part of a review paper† which Albert Sherman and $\mathrm{I}^{23}$ wrote in 1935 consisted in formulating and interpreting the secular problems connected with electrons in $s$ states in terms of the Dirac vector model.

\section{QUANTITATIVE SUCCESS WITH THE HYDROGEN MOLECULE}

Most of the results obtained in the early days of quantum mechanical valence theory were only qualitative in character. However, there were some exceptions where there were quantitative successes. One of these is the hydrogen molecule, so recalcitrant to calculation in the old quantum theory.

Table 1. Treatments of the ground state of $\mathrm{H}_{2}$

\begin{tabular}{lccc}
\hline \multicolumn{1}{c}{ Treatment } & $\begin{array}{c}\text { Max. binding } \\
\text { energy } \\
\mathrm{eV}\end{array}$ & $\begin{array}{c}\text { Equilibrium } \\
\text { separation } \\
\AA\end{array}$ & $\begin{array}{c}\text { Vibration } \\
\text { frequency } \\
\mathrm{cm}^{-1}\end{array}$ \\
\hline Heitler-London; done by Sugiura & $3 \cdot 13$ & 0.87 & $4 \cdot 8 \times 10^{3}$ \\
Molecular orbitals; Hylleraas & $3 \cdot 6$ & 0.70 & \\
Heitler-London with variable shielding; Wang & $3 \cdot 77$ & 0.73 & 4.9 \\
Wang + polarization; Rosen & $4 \cdot 02$ & 0.74 & 4.26 \\
Heitler-London + ionic term; Weinbaum & $3 \cdot 21$ & 0.90 & 4.75 \\
Wang + ionic; Weinbaum & $4 \cdot 00$ & 0.74 & \\
Wang + ionic + polarization; Weinbaum & $4 \cdot 10$ & - & \\
James and Coolidge, no $r_{12}$ & $4 \cdot 27$ & - & \\
James and Coolidge, with $r_{12}$ & $4 \cdot 70$ & 0.74 & 4.38 \\
Experiment & $4 \cdot 725 \pm 0.01$ & 0.74 & \\
\hline
\end{tabular}

$\dagger$ When I visited the U.S.S.R. in 1959 I was told that this paper had been translated into Russian. The Penney and Kynch article cited at $\S$ on previous page can be regarded as extending this review another two years. 


\section{J. H. VAN VLECK}

I reproduce here (as our Table 1) a table from the Van Vleck-Sherman article which shows that even by 1933 , very respectable quantitative precision had been obtained. The term 'ionic' means that the simple Heitler-London model is corrected to allow for the possibility of two electrons being on the same atom. The trial function of the Heitler-London type used by Wang ${ }^{24}$, which yielded moderately good results was exceedingly simple, viz.

$$
\psi=C\left[\exp \left\{-\alpha\left(r_{1 a}+r_{2 b}\right)\right\}+\exp \left\{-\alpha\left(r_{1 b}+r_{2 a}\right)\right\}\right]
$$

where $\alpha$ is a variable parameter and $r_{1 a}$ denotes the distance of electron 1 from nucleus a, etc. The entry 'no $r_{12}$ ' in Table 1 means that a molecular orbital type of calculation is made, without any attempt to allow for electron correlation by including in the arguments of the wave function the distance $r_{12}$ between the two electrons. James and Coolidge ${ }^{25}$ had only primitive, hand-cranked calculating devices but achieved remarkable agreement with experiment when dependence on $r_{12}$ is included.

\section{DIRECTED VALENCE-THE SLATER-PAULING MODEL}

A great advance in the Heitler-London method of approach for molecules other than $\mathrm{H}_{2}$ was made by Slater ${ }^{26}$ and Pauling ${ }^{27}$ in 1931 when they independently introduced the concept of directed valence. The crucial idea is that particular types of wave functions project out particularly in certain directions, like the horns on a cow. The Heitler-London-Slater-Pauling method is usually called that of the 'electron pair bond', but we prefer and shall use the term 'localized pair bond' to emphasize the fact that the electrons are regarded as confined to particular atoms with paths (except for $s$ states) projecting in particular directions. This distinction seems advisable since electron pairs are also involved in molecular orbital theory, inasmuch as two electrons can nestle in a given orbital state. The simplest example of directed valence is provided by $p$ wave functions. If instead of using the forms with exponential angular factors corresponding to the quantization of angular momentum one takes the wave functions to be real, then one has three mutually orthogonal functions of the form $\mathrm{f}(r) x, \mathrm{f}(r) y, \mathrm{f}(r) z$, whose corresponding charge density clouds are dumb-bell shaped with mutually perpendicular axes. One thus understands why the ammonia molecule has a pyramidal structure, and the water molecule a bent one in which the two $\mathrm{O}-\mathrm{H}$ axes are not too far from a right angle. With a non-directional, $s$-state type of theory the repulsions between the hydrogen atoms would presumably control the type of arrangement, and ammonia would be plane and the water molecule linear and non-polar.

To explain the tetrahedral structure of methane, the by now well-known concept of $s p$ hybridization must be introduced, thereby obtaining wave functions projecting in the four tetrahedral directions.

The structure of benzene is interpreted as follows. If the $x, y$ plane be that of the benzene ring, then by hybridization of $s, p_{x}$ and $p_{y}$ wave functions each carbon atom has a single bond to a hydrogen and to each of its neighbours with angles of $120^{\circ}$ between these bonds. In addition there are the exchange energies connecting the $p_{z}$ wave functions, the so-called $\pi-\pi$ bonds. 


\section{RESONANCE ENERGIES}

The $\pi-\pi$ electron pair bonds in benzene can be drawn in five different ways as shown in Figure 2. The true wave function is a linear combination of all five structures, and the total bonding energy is greater than if one structure alone, such as A, were utilized. In other words the benzene molecule resonates through a variety of configurations and the corresponding increase in bonding energy is called the resonance energy. Calculation shows that if $W$ denotes the $\pi-\pi$ exchange energy computed without resonance (configuration $\mathrm{A}$ or $\mathrm{B}$ ), then if resonano between $\mathrm{A}$ and $\mathrm{B}$ is included it becomes $1.6 \mathrm{~W}$ and $1.74 \mathrm{~W}$ if $\mathrm{C}, \mathrm{D}$ and $\mathrm{E}$ are included as well ${ }^{28}$. The concept, and more

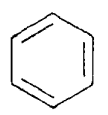

A

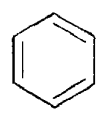

B

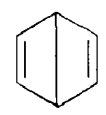

C

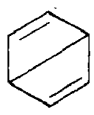

D

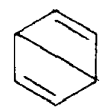

$E$

Figure 2. Different constituent bond pairs in benzene.

or less quantitative calculation of resonance energies, is one of the early triumphs of quantum chemistry, something that could not even be qualitatively described without the language of quantum mechanics. The energies of a number of hydrocarbon molecules can be calculated surprisingly well by a simple, Heitler-London type of theory if the parameters (the exchange integrals) are determined partly spectroscopically, partly by fitting the observed bonding energies of certain molecules (those in parenthesis in Table 2). This procedure was utilized in an early paper by Serber ${ }^{29}$. His results are shown in Table 2. The agreement with experiment is better than one has any right to expect in view of the primitive character of the calculations. If the effect of resonance is omitted, the agreement is not as good.

Table 2. Energies of hydrocarbons

\begin{tabular}{llcc}
\hline Hydrocarbon & & $\begin{array}{c}\text { Calc. } \\
\mathrm{eV}\end{array}$ & $\begin{array}{c}\text { Obs. } \\
\mathrm{eV}\end{array}$ \\
\hline Methane & $\mathrm{CH}_{4}$ & $(16 \cdot 3)$ & $16 \cdot 3$ \\
Ethane & $\mathrm{C}_{2} \mathrm{H}_{6}$ & $(27 \cdot 6)$ & $27 \cdot 6$ \\
Propane & $\mathrm{C}_{3} \mathbf{H}_{8}$ & $38 \cdot 9$ & $38 \cdot 9$ \\
Butane & $\mathrm{C}_{4} \mathbf{H}_{10}$ & $50 \cdot 2$ & $50 \cdot 2$ \\
Ethylene & $\mathrm{C}_{2} \mathbf{H}_{4}$ & $(21 \cdot 8)$ & $21 \cdot 8$ \\
Propylene & $\mathrm{C}_{3} \mathbf{H}_{6}$ & $33 \cdot 1$ & $33 \cdot 1$ \\
1,2,3,4-Tetrahydrobenzol & $\mathrm{C}_{6} \mathbf{H}_{10}$ & $61 \cdot 9$ & $61 \cdot 8$ \\
Cyclohexane & $\mathrm{C}_{6} \mathbf{H}_{12}$ & $67 \cdot 4$ & $67 \cdot 1$ \\
\hline
\end{tabular}

Resonance energies can also be calculated in a rather different fashion by the method of molecular orbitals, as first done by Hückel ${ }^{30}$ and the agreement of the two types of calculation is usually surprisingly good. For instance, both give 1.84 for the ratio of the resonance energy of naphthalene to that of benzene ${ }^{31}$. The so-called experimental value (determined by departure from bond additivity) is 1.90 . No doubt this rather fortuitous agreement would be spoiled if attempts are made to make the calculations a little better by correcting for non-orthogonality or electron repulsion. Many 
of the early calculations seemed to possess the property that they worked best in over-simplified form, although of course a really precise calculation must yield the right answer.

\section{DIFFICULTIES IN BOTH METHODS}

The picture which we have presented so far is excessively rosy. Both the localized pair bond and molecular orbital approaches had their particular successes and difficulties. A great triumph of the method of molecular orbitals is its prediction that the oxygen molecule should be paramagnetic, something not at all obvious with the localized bond. The great sin of the method of molecular orbitals is the omission of the perturbing effect of inter-electron repulsion. In consequence elementary schematic calculations with molecular orbitals predict an excessive affinity for extra atoms, giving, for example, a lower energy for $\mathrm{H}_{3}$ than $\mathrm{H}_{2}+\mathrm{H}$, as Coulson ${ }^{32}$ pointed out over thirty years ago. The great sin of the localized bond approach is neglecting non-orthogonality corrections but at the same time assuming the exchange integrals to be negative, a sign behaviour necessary for an electron pair bond to correspond to anti-parallel rather than parallel spins. With orthogonal wave functions the integrals are always positive, a reflection of the Hund rule in atomic spectra. Of course all the difficulties must disappear when the calculations are made with enough refinement and proper corrections. For example one can try using the Heitler-London method with orthogonalized wave functions, but then the exchange integrals are positive, and then it is absolutely essential to include polarization corrections, or in other words inter-configuration interaction. Under these conditions the method loses all simplicity and it would be better to employ molecular orbitals.

\section{COMFORTING AGREEMENT BETWEEN BOTH METHODS}

It is instructive to compare the predictions made by the molecular orbital and localized pair approaches. The good agreement in the matter of resonance energies has already been mentioned. When I collaborated with Dr Albert Sherman I remember him remarking that the thing he had learned most of all from me was the advisability of making a calculation in more than one way. A concluding paragraph of the review article ${ }^{23}$ which we wrote together read in part as follows: 'Since molecular orbitals and the H-L-S-P procedures represent two different types of approximation, neither any too good, it is clear that much more confidence can be placed in the results of the two methods when they agree than can otherwise. If certain properties are found to be true under these two different kinds of approximation, warranted under different idealized limiting conditions, it is natural to suppose that the same properties are also valid in the actual, more complicated intermediate case. It is therefore a comfort that both theories predict a nearly right-angled model for water, a tetrahedron for methane, etc.'

In the 1930s I wrote a number of papers ${ }^{33}$ endeavouring to correlate qualitatively the two types of approach, including their inter-relation ${ }^{34,35}$ in terms of group theory, and to show that they would yield similar predictions regarding geometrical arrangement, with particular emphasis on $\mathrm{CH}_{4}$ and 
molecules closely related thereto. I'm afraid that I didn't take these calculations too seriously and I didn't pursue them after 1935, partly because my background in chemistry was shaky and partly because the calculations were so crude in nature, and I didn't realize they would attract as much attention as they did. Also, above all, my research interests were attracted towards the crystalline field theory of the magnetic properties in the iron group, and I will devote the balance of my talk to it. This subject is particularly appealing because, thanks to group theory, one can get almost quantitative results out of purely qualitative considerations.

\section{CRYSTALLINE FIELD THEORY AND THE PARAMAGNETISM OF IRON GROUP COMPOUNDS}

Crystalline field theory has provided a rational interpretation of the phenomena of coordination chemistry of the transition ions. However, historically this development came about for the most part not by direct frontal attack on chemical problems, but as a byproduct of the study of paramagnetism by theoretical physicists.

The crystalline field concept is an exceedingly simple one. The paramagnetic ion is regarded as exposed in the solid state to a crystalline potential having the appropriate symmetry. If charge transfer is neglected the general crystalline potential has the form

$$
V(x, y, z)=\Sigma_{n, m} a_{n m} r^{n} Y_{n}^{m}(\theta, \varphi)
$$

where the $Y_{n}^{m}$ are spherical harmonics. The maximum values of $n$ can be shown to be four and six respectively for $d$ and $f$ electrons. In the important case of $d$ electrons in an accurately cubic field expression 6 can be reduced to a single member.

$$
V=D\left(x^{4}+y^{4}+z^{4}-\frac{3}{5} r^{4}\right)
$$

Crystalline field theory has been extensively applied both to iron group and rare earth compounds. I shall omit discussing the rare earths, for two reasons. In the first place, during the 1920 s and 1930 s they were regarded as rather esoteric materials whereas today they are important for such engineering devices as lasers and colour television. Secondly, the crystalline potentials acting on the $4 f$ electrons are too small to account for much of any chemical binding, although it should not be overlooked that the paramagnetism of these electrons serves as a valuable indicator of what the valence is, and the degree of chemical purification as well as in recent years, the nature of the lattice site.

I think most readers are familiar with the fact that salts of the iron group have susceptibilities quite different from what one would calculate for free ions, and that a sufficiently symmetrical field will quench the orbital angular momentum, leaving the spin free, so that the susceptibility conforms to the spin-only value given in our equation 1 .

It is, however, the deviations from the spin-only values that are of particular interest, and especially the anisotropy, which is markedly different for different salts, as shown in Table 3 where some early experimental data are exhibited. 


\section{J. H. VAN VLECK}

Table 3. Magnetic anisotropy

\begin{tabular}{|c|c|c|c|}
\hline Ion & State & Salt measured & $\begin{array}{c}\text { Anisotropy } \\
\%\end{array}$ \\
\hline $\mathrm{Mn}^{2+}$ & $d^{5}{ }^{6} S$ & $\mathrm{Mn}\left(\mathrm{NH}_{4}\right)_{2}\left(\mathrm{SO}_{4}\right)_{2} \cdot 6 \mathrm{H}_{2} \mathrm{O}$ & $0 \cdot 10$ \\
\hline $\mathrm{Fe}^{3+}$ & $d^{5}{ }^{6} S$ & $\mathrm{FeK}_{3}\left(\mathrm{C}_{2} \mathrm{O}_{4}\right)_{3} \cdot 3 \mathrm{H}_{2} \mathrm{O}$ & 0.20 \\
\hline $\mathrm{Fe}^{2+}$ & $d^{6}{ }^{5} \mathrm{D}$ & $\mathrm{FeK}_{2}\left(\mathrm{SO}_{4}\right)_{2} \cdot 6 \mathrm{H}_{2} \mathrm{O}$ & 16 \\
\hline $\mathrm{Co}^{2+}$ & $d^{7}{ }^{4} F$ & $\mathrm{Co}\left(\mathrm{NH}_{4}\right)_{2}\left(\mathrm{SO}_{4}\right)_{2} \cdot 6 \mathrm{H}_{2} \mathrm{O}$ & 30 \\
\hline $\mathrm{Ni}^{2+}$ & $d^{83} F$ & $\mathrm{Ni}\left(\mathrm{NH}_{4}\right)_{2}\left(\mathrm{SO}_{4}\right)_{2} \cdot 6 \mathrm{H}_{2} \mathrm{O}$ & 1.5 \\
\hline $\mathrm{Cu}^{2+}$ & $d^{9}{ }^{2} F$ & $\mathrm{Cu}\left(\mathrm{NH}_{4}\right)_{2}\left(\mathrm{SO}_{4}\right)_{2} \cdot 6 \mathrm{H}_{2} \mathrm{O}$ & 20 \\
\hline
\end{tabular}

It is astonishing, at first sight, that the anisotropy should be so different for cobalt and nickel, as both ions are in $F$ states.

The answer is to be found in assuming a crystalline field that is nearly cubic. One can show by group theory as first developed by Bethe ${ }^{36}$ for crystals or otherwise, that an $F$ state will decompose in the fashion shown in Figure 3. If a non-degenerate level is deepest, then the orbital moment is completely quenched*, and there should be almost complete isotropy. On the other hand, if Figure 3 is upside down, and if the components $a, b, c$ of the ground level do not coincide because of deviations from cubic symmetry, and so have different Boltzmann factors, the anisotropy will be considerable.

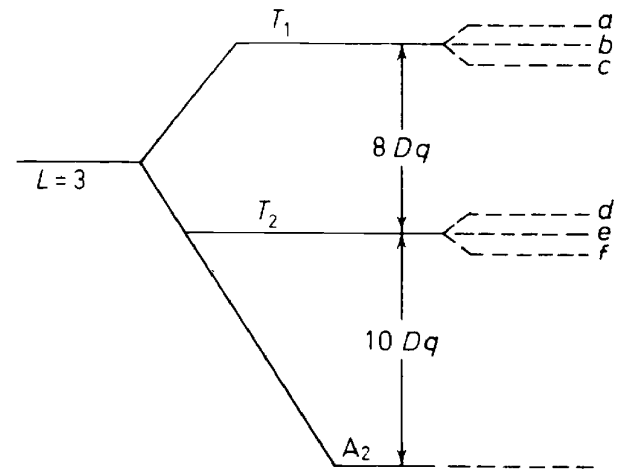

Figure 3. Orbital energies of an $F$ state in a cubic crystalline field. The further decompositions $\mathrm{a}-\mathrm{b}-\mathrm{c}$ or $\mathrm{d}-\mathrm{e}-\mathrm{f}$ ensue only if there are small deviations from cubic symmetry. The quantity $D q$. is connected with the constant $D$ of equation 7 by the relation $D q=2 D\left\langle r^{4}\right\rangle_{\lambda v} / 105$.

We therefore see that the very different behaviour of nickel and cobalt can be explained if it is assumed that Figure 3 is properly placed for $\mathrm{Ni}^{2+}$, but should be upside down in $\mathrm{Co}^{2+}$. Detailed calculations pioneered by Schlapp

* Figures 3 and 5 do not show the additional multiplicity of energy levels when spin is included. For instance, the ground level $\mathrm{A}_{2}$ of $\mathrm{Ni}^{2+}$ in a cubic field becomes instead a triply degenerate level when spin is included. However, the splitting of the components by a non-cubic potential is a very small one (useful incidentally for constructing solid state masers) so that at room temperatures the spin-only formula is obeyed almost perfectly. 


\section{SPIN, THE GREAT INDICATOR OF VALENCE BEHAVIOUR}

and Penney ${ }^{37}$ show that then good agreement can be obtained with experiment, and over the years the computations have been extended and refined, in large part by the Japanese.

The question now arises as to whether this inversion of Fiqure 3 in going from $\mathrm{Ni}^{2+}$ to $\mathrm{Co}^{2+}$ is an honest procedure, for the crystalline potential cannot differ materially for atoms adjacent in the periodic table such as $\mathrm{Ni}$ and Co. For a while this worried Schlapp, Penney and myself in 1932. Then one day it dawned on me that a simple calculation ${ }^{38}$ based on the invariance of the diagonal sum shows that the splitting pattern does indeed invert in going from nickel to cobalt even though the constant $D$ in equation 7 remains the same. I will not go into the details of the analysis, which is very similar to that used by Goudsmit in showing that multiplets invert in going from the left to the right half of an unfilled shell.

At this stage the reader may feel that the theory which I have described is a fine thing for magnetism, but has no particular relevance for valence behaviour and chemical bonding. However, this is not true. I believe that the splitting of the energy levels in nearly cubic crystalline fields, and particularly their inversion properties which I first deduced in my favourite paper ${ }^{38}$, written in 1932, furnished the clue to the correct understanding and interpretation of the coordination properties of elements of the transition group. I have so far discussed only the relative sign of the splittings, e.g. if Figure 3 is upright in $\mathrm{Ni}^{2+}$ it should be inverted in $\mathrm{Co}^{2+}$, but why not vice versa? In 1932 Gorter ${ }^{39}$ answered this question by computing the form of the potential to be expected from the anions surrounding the paramagnetic cation. He treated the anions as simple point charges (or equally well, radially directed dipoles), an approximation quite adequate to answer the question of the sign of $D$ in equation 7 . He found that if there are six anions octahedrally arranged then Figure 3 should be upright in $\mathrm{Ni}^{2+}$ rather than in $\mathrm{Co}^{2+}$. The magnetic compounds listed in Table 3 are indeed of a type where the coordination is known to be sixfold. If, on the other hand, the coordination is four, as for a tetrahedral complex, the situation is reversed, i.e. Figure 3 is upright in $\mathrm{Co}^{2+}$ rather than in $\mathrm{Ni}^{2+}$. Consequently one should expect tetrahedrally coordinated cobalt to be nearly isotropic magnetically. Soon after this was pointed out, Krishnan and Mookherji ${ }^{40}$ in Delhi prepared two compounds, $\mathrm{Cs}_{3} \mathrm{CoCl}_{5}$ and $\mathrm{Cs}_{2} \mathrm{CoCl}_{4}$, which are known to have fourfold coordination, and they did indeed find low anisotropies, respectively $6 \frac{1}{2}$ and 5 per cent as compared with about 30 per cent for six-coordinated cobalt.

So far I have cited examples where the coordination and geometrical arrangement were known by the chemists without resort to the magnetic data. It is, however, possible to invert the procedure and use the magnetic properties to deduce the otherwise unknown type of coordination. This has become a powerful tool in the inorganic chemistry of the transition elements in modern times though there was little interest in the subject in the 1930s. An early example can, however, be found in the case of $\mathrm{CuSO}_{4} \cdot 5 \mathrm{H}_{2} \mathrm{O}$. From the magnetic properties Jordah ${ }^{41}$ and I argued in 1933-34 that the coordination of the anions around the $\mathrm{Cu}$ had to be 6-rather than 5-fold. This, however, was not accepted by the crystallographers and chemists, as there were only five waters of hydration per $\mathrm{Cu}^{2+}$ ion, and one can't produce six 


\section{J. H. VAN VLECK}

things from five objects. However, $\mathrm{x}$-ray measurements by Beevers and Lipton ${ }^{42}$ in England soon showed that there were indeed six oxygen ions around each $\mathrm{Cu}^{2+}$ ion. The point is that the crystalline structure is such that certain $\mathrm{O}^{2-}$ ions do double duty as being neighbouring anions for two $\mathrm{Cu}^{2+}$ ions.

\section{EXPLANATION OF IRREGULARITIES IN HEATS OF SOLUTION}

A rather interesting corollary of the crystalline field theory is concerned with the heats of formation of various divalent ions in solutions. In going from ion to ion a strange looking curve is obtained, the upper one in Figure 4.

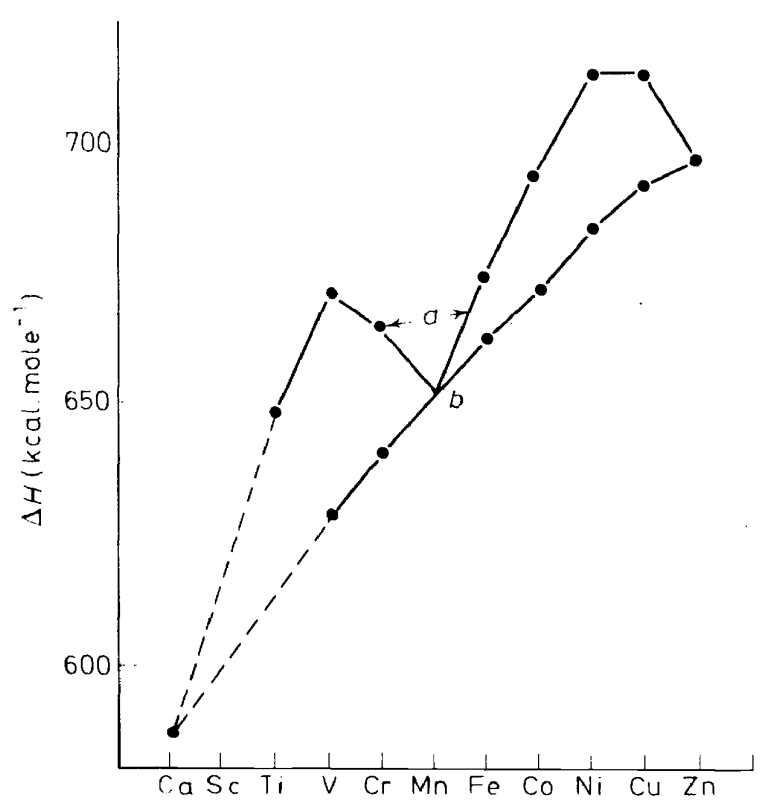

Figure 4. Heats of formation of divalent aqueous ions, taken from $\mathrm{p} 316$ of Griffith's The Theory of Transition Metal Ions, and based on work by Orgel.

The explanation of the anomaly was first given by Penney ${ }^{43}$ in 1940 , and rediscovered by Orgel ${ }^{44}$ in 1952 . It is that around the paramagnetic ion in solution one has six waters of coordination. If one averages over all the Stark components the splitting is zero, but if the splitting is large compared to $k T$, only the lowest components are inhabited. There is consequently an extra lowering or heat of solution. For example, in Figure 3, $A_{2}$ is deeper than the unsplit level $L=3$. For manganese, which has a half filled $d$ shell and is in an $S$ state, there is no such lowering. For the other ions one can estimate how much this $\mathrm{x}$-ray Stark stabilization amounts to, using substantially the Stark splittings employed in solids with six waters of coordination. When one does this, one gets a smooth curve (the lower one of Figure 4). 


\section{COLOUR}

The characteristic colours as well as the magnetic properties of the different ions of the transition group are part and parcel of crystalline field theory. The absorption bands responsible for the colours of the different ions are are due to transitions between the different levels such as are shown in Figure 3. Space will not permit me to describe how the crystal field theory explains how octa-coordinated cobalt salts are a weak pink, tetra-coordinated ones a brilliant blue, octa-coordinated nickel green, etc. All this can be done in terms of a single parameter $D q$ which does not vary drastically from ion to ion, or from compound to compound as shown in Table 4 (only $2+$ or $3+$ ions should be compared $\dagger$. The similarity of the values of $D q$ in solution

Table 4. Values of $D q$ in $\mathrm{cm}^{-1}$

\begin{tabular}{|c|c|c|c|c|c|}
\hline & & $6 \mathrm{Br}^{-}$ & $6 \mathrm{Cl}^{-}$ & $6 \mathrm{H}_{2} \mathrm{O}$ & $6 \mathrm{NH}_{3}$ \\
\hline $3 d^{1}$ & $\mathrm{Ti}^{3+}$ & & & 2030 & \\
\hline $3 d^{2}$ & $\mathrm{~V}^{3+}$ & & & 1800 & \\
\hline \multirow[t]{2}{*}{$3 d^{3}$} & $\mathrm{~V}^{2+}$ & & & 1180 & \\
\hline & $\mathrm{Cr}^{3+}$ & & 1330 & 1760 & 2160 \\
\hline $4 d^{3}$ & $\mathrm{Mo}^{3+}$ & & 1920 & & \\
\hline \multirow[t]{2}{*}{$3 d^{4}$} & $\mathrm{Cr}^{2+}$ & & & 1400 & \\
\hline & $\mathrm{Mn}^{3+}$ & & & 2100 & \\
\hline \multirow[t]{2}{*}{$3 d^{5}$} & $\mathrm{Mn}^{2+}$ & & & 750 & \\
\hline & $\mathrm{Fe}^{3+}$ & & & 1400 & \\
\hline \multirow[t]{2}{*}{$3 d^{6}$} & $\mathrm{Fe}^{2+}$ & & & 1000 & \\
\hline & $\mathrm{Co}^{3+}$ & & & 1910 & \\
\hline $3 d^{7}$ & $\mathrm{Co}^{2+}$ & & & 1000 & 1050 \\
\hline $3 d^{8}$ & $\mathrm{Ni}^{2+}$ & 600 & & 860 & 1080 \\
\hline $3 d^{9}$ & $\mathrm{Cu}^{2+}$ & & 650 & 1260 & 1510 \\
\hline
\end{tabular}

and in the solid state is an illustration of the fact that the local order in liquids is much more like that in solids than was deemed to be the case fifty years ago. Most of the work on the spectroscopy of the energy levels is post-1945. There was little interest in the subject in the 1930s. When I went to a conference of the Faraday Society in Dublin on transition elements in 1958, it was an eye-opener to me to find physical chemists continually talking about the parameter $D q$.

\section{THE LOW VERSUS HIGH SPIN CASES}

I have so far assumed that the crystalline field is not powerful enough to destroy Russell-Saunders coupling, so that $L, S$ are good quantum numbers. However, in strong fields the ions may want to double up in states of low crystalline Stark energy in order to keep their Stark energy low. This is what chemists call the strong field case, to distinguish from the one which I have discussed, which is called the high spin or weak field one, but I consider the

$\uparrow$ Table 4 is taken from a review article by D. S. MClure in Solid State Physics, 9, 426 (1959) and is based on work of Jørgenson and of MClure. Other references on colour, etc., will be found in this article. 


\section{J. H. VAN VLECK}

term weak field a misnomer for a field that can give splittings measured in volts. The splitting to be expected for a single $d$ electron in an octahedral field is shown in Figure 5. In the hydrated sulphates, the customary high spin case, the spins of the five $d$ electrons of $\mathrm{Fe}^{3+}$ have all to be parallel to achieve the maximum spin $S=5 / 2$ demanded by the Hund rule. The five orbital states are then equally populated, giving a centrosymmetric or $S$ state (cf. our equation 2). The susceptibility is then highly isotropic and conforms

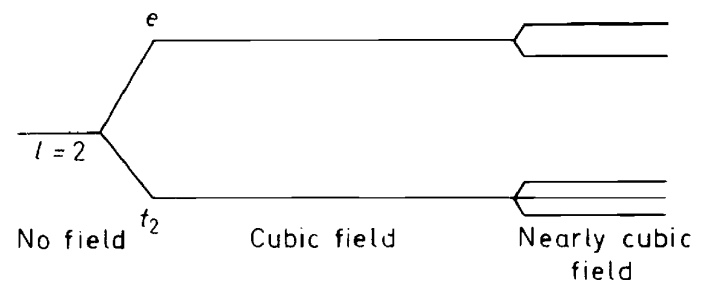

Figure 5. Orbital energies of a single $d$ electron. If the symmetry is tetrahedral rather than octahedral, the figures should be inverted.

closely to the spin-only value given in equation 1 with $S=5 / 2$. However, if the splitting of energy levels caused by the crystalline Stark effect is sufficiently great, the Hund rule is sacrificed and two of the three deep cubic states are doubly occupied, and only the last one has a state to itself. The spin is then $1 / 2$. This case is realized experimentally in the ferric cyanides. The susceptibility is, however, not given by a formula as simple as our equation 1 with $S=1 / 2$, as the orbital moment of the fifth electron is not quenched. Similarly, in the ferrous cyanides, all six $d$ electrons of the $\mathrm{Fe}^{2+}$ ions prefer to settle in the three low levels of Figure 5, giving a resultant spin $S=0$ instead of the value $S=2$ characteristic of the Hund rule. It is indeed found experimentally that $\mathrm{K}_{4} \mathrm{Fe}(\mathrm{CN})_{6}$ is diamagnetic. Magnetic measurements have even been used ever since the 1930 s beginning with Pauling and Coryel1 ${ }^{45}$ to study the chemical behaviour of iron in blood. For example, the ferrohaemoglobin ion exhibits high and low spin values, 2 and 0 in the presence of $\mathrm{H}_{2} \mathrm{O}$ and $\mathrm{O}_{2}$ molecules respectively.

At this point I must mention that the distinction between the low and high spin cases was first enunciated by Pauling ${ }^{46}$. However, he drew his conclusions on the basis of highly directed wave functions for the transition ion constructed by hybridization of $3 d, 4 s$ and $4 p$ wave functions, whereas I used crystal field theory ${ }^{47}$. In our opinion Pauling's model is not very realistic, as it requires the paramagnetic ion to be negatively charged, $\mathrm{Fe}^{3-}$ rather than the conventional $\mathrm{Fe}^{3+}$. That Pauling reached correct conclusions is a tribute to the brilliance of his chemical intuition, and once again illustrates the statement made at the beginning of this paper that a really good chemist could get right answers with wrong models. However, it should at the same time be remarked that a complete wave function is presumably a linear combination of everything in sight, and so may contain some terms where the paramagnetic ion behaves like an anion rather than cation. In general 
it can be shown ${ }^{47}$ that rather similar conclusions regarding the coordination and magnetic properties of the transition ions can be obtained with three different models, viz. the localized pair bond theory of Pauling based on hybridization, crystalline field theory and the method of molecular orbitals.

\section{COVALENCY AND LIGAND FIELD THEORY}

So far I have discussed simple crystalline field theory in which electrons are regarded as localized on particular atoms. I want now to pass to the more powerful theory in which the model is generalized to allow for the ambulatory properties of electrons or, in other words, for at least partial covalency, a subject very dear to the heart of the chemist. This generalized crystalline field theory where the wandering tendencies of electrons are allowed for by the method of molecular orbitals is called ligand field theory. The method was developed qualitatively in the $1930 \mathrm{~s}^{47}$. It has great flexibility and allows for any degree of covalency. It is outside the scope of the present historical paper to discuss in any detail how the degree of covalency involved in the molecular orbital approach has been studied quantitatively in recent years by magnetic resonance studies of hyperfine structure. By such measurements Tinkham ${ }^{48}$, for instance, finds that when $\mathrm{Mn}^{2+}$ is doped into $\mathrm{ZnF}_{2}$, the electrons responsible for the paramagnetism of $\mathrm{Mn}^{2+}$ spend only 60 per cent of the time on the $\mathrm{Mn}$ ion and the rest of the time on the surrounding ligands. That the covalency rather than electrostatic effects may be responsible for the bonds involving $d$ electrons was emphasized as early as 1932 by Pauling ${ }^{49}$. He pointed out that of all the ligands, the fluourine ones are those involving the most complete polarity, i.e. closest to the completed shell structure $\mathrm{F}^{-}$, and yet the fluourine compounds actually show the high rather than low spin behaviour.

\section{THE JAHN-TELLER EFFECT}

Another complication besides covalence which came to light in the 1930s is the Jahn-Teller effect. Jahn and Teller ${ }^{50}$ showed that in general when a state is degenerate because of a high degree of spatial symmetry, the energy will be lowered if the molecule (or crystal as the case may be) is warped. This is a highly mathematical and difficult subject. One even distinguishes between the static and dynamic Jahn-Teller effects according as the warping is more or less stationary or resonating through a variety of configurations. At first the Jahn-Teller effect was considered only rather esoteric physics, but has been taken over by the chemists because of the light it throws on reduction in $g$-factors, irregularities in interatomic spacings, etc. The appearance of the paper by Jahn and Teller in 1937 may be regarded as marking the end of what I may call the conceptual period of the quantum mechanics of valence, as later developments have been in matters of detail rather than fundamental new ideas, and therefore seems an appropriate end-point for my survey.

\section{CONCLUSION}

It would be gratifying if one could calculate from pure theory the size of the crystalline field and amount of covalency, but attempts to do this have 


\section{J. H. VAN VLECK}

had little success even today with modern computing machinery. Consequently Freeman and Watson ${ }^{51}$ are led to remark: "crystal field theory has never been theoretically justified in that although in principle $D q$ can be determined theoretically within the framework of the theory no accurate a priori determinations of the crystal field strength have in fact been made'. Nevertheless one cannot deny that the parameter $D q$ had deep physical and chemical meaning even in the 1930s, even though it cannot be calculated from scratch. The reason is that it has a general basis in group theory not dependent on the details of the model. Such things as magnetic susceptibilities, colour, degree of covalency, variations from ion to ion in the energy of stabilization in solution, strength of bonding geometry of local arrangements and incipient covalency cease to be mysteries. In the words of the late Professor Moffitt ${ }^{52}$, written two years before his untimely death in 1958: 'It will be a long time before a method is developed to surpass in simplicity, elegance and power that of crystal field theory. Within its extensive domain it has provided at very least a deep qualitative insight into the behaviour of a many-electron system. No other molecular theory, to our knowledge, has provided so many useful numbers which are so nearly correct. And none has a better immediate prospect of extending its chemical applications.'

\section{REFERENCES}

1 I. Langmuir, J. Amer. Chem. Soc. 41, 868 (1919); also notable earlier papers by G. N. Lewis, J. Amer. Chem. Soc. 38, 762 (1916) and W. Kossel, Ann. Phys. Lpz. 49, 229 (1916).

2 N. Bohr, Essay 3 of the Theory of Spectra and Atomic Constitution. Cambridge University Press: London (1922); also published in Danish in 1921 and in German in Z. Phys. 9, 1 (1922)

3 I. Langmuir, Colloid Chemistry, 1, 525 (1926).

4 J. D. Main Smith, Chemistry and Atomic Structure, Van Nostrand: New York (1924).

5 I. Langmuir, Science, 53, 290 (1921).

6 I. Langmuir, Phys. Rev. 17, 339 (1921); Science, 52, 434 (1920).

7 W. Pauli, Z. Phys. 31, 765 (1925).

8 G. E. Uhlenbeck and S. Goudsmit; Naturwissenschaften, 13, 953 (1925); Nature, Lond. 117, 264 (1926).

9 J. H. Van Vleck, 'Quantum principles and line spectra'. Bulletin No. 54 of the National Research Council.

10 E. C. Stoner, Phil. Mag. 48, 719 (1924).

11 A. Landé, Z. Phys. 16, 391 (1923).

12 L. H. Thomas, Nature, Lond. 117, 514 (1926); Phil. Mag. 3, 1 (1927).

13 P. A. M. Dirac, Proc. Roy. Soc. A, 123, 714 (1929).

14 P. A. M. Dirac, Proc. Roy. Soc. A, 117, 610 (1928); 118, 351 (1928).

15 W. Heisenberg, Z. Phys. 38, 411 (1926).

P. A. M. Dirac, Proc. Roy. Soc. A, 112, 661 (1926).

16 G. C. Havens, Phys. Rev. 41, 337 (1932).

17 F. H. Verhoek and F. Daniels, J. Amer. Chem. Soc. 53, 1250 (1931).

18 P. Pershan, R. G. Shulman, D. J. Wyluda and J. Eisinger, Physics, 1, 163 (1934).

19 H. J. Ewen and E. M. Purcell, Nature, Lond. 168, 356 (1951).

20 W. Heitler and F. London, Z. Phys. 44, 455 (1927); for other references, including those for the calculations listed in our Table 1, see the review article cited in ref. 23.

21 W. Heitler, Z. Phys. 46, 47 (1928); 47, 835 (1928); 51, 805 (1929).

22 M. Born, Z. Phys. 64, 729 (1930); 65, 718 (1930).

23 J. H. Van Vleck and A. Sherman, Rev. Mod. Phys. 7, 167 (1935).

24 S. C. Wang, Phys. Rev. 31, 579 (1928).

25 H. M. James and A. S. Coolidge, J. Chem. Phys. 1, 825 (1933).

26 J. C. Slater, Phys. Rev. 37, 481 (1931); 38, 1109 (1931).

27 L. Pauling, J. Amer. Chem. Soc. 53, 1367 (1931); 54, 988 and 3370 (1932). 


\section{SPIN, THE GREAT INDICATOR OF VALENCE BEHAVIOUR}

${ }^{28}$ L. Pauling and G. W. Wheland, J. Chem. Phys. 1, 362 (1933): also E. Hückel, Z. Phy. 70, 204 (1931).

29 R. Serber, J. Chem. Phys. 3, 81 (1935).

30 E. Hückel, Z. Phys. 60, 423 (1929).

31 For an early paper comparing the results of calculating resonanœe energies with the various methods see G. W. Wheland, J. Chem. Phys. 2, 474 (1934).

32 C. A. Coulson, Proc. Camb. Phil. Soc. 31, 244 (1935).

33 J. H. Van Vleck, J. Chem. Phys. 1, 177 and 219 (1933); 2, 20 and 297 (1934).

34 J. H. Van Vleck, J. Chem. Phys. 3, 803 (1935). A paper of practically the same content as mine on the group theory relations between the two methods was published by another author ${ }^{35}$ in the same journal five years later. That this happened is a testimonial of how hard it was for authors, referees, and editors to keep abreast of the literature even in the early days.

${ }^{35}$ G. E. Kimball, J. Chem. Phys. 8, 188 (1940).

${ }^{36}$ H. Bethe, Ann. Phys. Lpz. 3, 133 (1929).

37 R. Schlapp and W. G. Penney, Phys. Rev. 42, 666 (1932).

38 J. H. Van Vleck, Phys. Rev. 41, 208 (1932).

39 C. J. Gorter, Phys. Rev. 42, 437 (1932).

40 K. S. Krishnan and A. Mookherji, Phys. Rev. 51, 528 and 774 (1937).

41 O. Jordahl, Phys. Rev. 45, 87 (1934); 46, 79 (1934).

42 C. A. Beevers and H. Lipson, Proc. Roy. Soc. 146, 570 (1934).

43 W. G. Penney, Trans. Faraday Soc. 36, 627 (1940).

44 L. E. Orgel, J. Chem Soc. (London), 4756 (1952).

45 L. Pauling and C. D. Coryell, Proc. Nat. Acad. Sci. Wash. 22, 159 and 210 (1936).

${ }^{46}$ L. Pauling, J. Amer. Chem. Soc. 53, 1367 (1931).

47 J. H. Van Vleck, J. Chem. Phys. 3, 807 (1935).

48 M. Tinkham, Proc. Roy. Soc. A, 236, 535 and 549 (1956).

49 L. Pauling, J. Amer. Chem. Soc. 54, 988 (1932).

50 H. A. Jahn and E. Teller, Proc. Roy. Soc. 161, 220 (1937).

51 A. J. Freeman and R. E. Watson, Phys. Rev. 120, 254 (1960).

52 W. Moffitt and C. J. Ballhausen, Ann. Rev. Phys. Chem. 7, 107 (1956). 